\title{
BMJ Open Effect of adiposity on differences in carotid plaque burden in studies conducted in Norway and Russia: a cross-sectional analysis of two populations at very different risk of cardiovascular mortality
}

Yume Imahori (1) , ${ }^{1}$ Chris Frost, ${ }^{1}$ Ellisiv B Mathiesen, ${ }^{2}$ Andrey Ryabikov, ${ }^{3,4}$ Alexander V Kudryavtsev, ${ }^{5}$ Sofia Malyutina, ${ }^{3,4}$ Michael Kornev, ${ }^{5}$ Alun D Hughes, ${ }^{6}$ Laila A Hopstock, ${ }^{2}$ David A Leon ${ }^{1,2}$

To cite: Imahori Y, Frost C, Mathiesen EB, et al. Effect of adiposity on differences in carotid plaque burden in studies conducted in Norway and Russia: a crosssectional analysis of two populations at very different risk of cardiovascular mortality. BMJ Open 2020;10:e036583. doi:10.1136/ bmjopen-2019-036583

- Prepublication history and additional material for this paper are available online. To view these files, please visit the journal online (http://dx.doi. org/10.1136/bmjopen-2019036583).

Received 27 December 2019 Revised 15 March 2020 Accepted 31 March 2020
Check for updates

(C) Author(s) (or their employer(s)) 2020. Re-use permitted under CC BY-NC. No commercial re-use. See rights and permissions. Published by BMJ.

For numbered affiliations see end of article.

Correspondence to Dr Yume Imahori; yumeim0405@gmail.com

\section{ABSTRACT}

Objectives Large differences exist in the burden of cardiovascular disease (CVD) between Russia and Western European countries including Norway. Obesity prevalence may contribute to the differences. We investigated whether difference in the level of adiposity, assessed using body mass index and waist-to-hip ratio(WHR), could explain intercountry differences in the burden of carotid plaque, a measure of atherosclerosis, in the populations.

Design Cross-sectional analysis. Logistic and linear regression models were used.

Setting We used population-based cross-sectional Know Your Heart (KYH) study in Russia and the Troms $\emptyset 7$ study (Tromsø 7) in Norway.

Participants 3262 and 1800 men and women aged 40-69 years in KYH and Troms $\emptyset$ 7, respectively.

Primary and secondary outcome The presence of carotid plaques and plaque score assessed using ultrasound.

Results The presence of carotid plaques and plaque score were higher in KYH than Troms $\emptyset$ regardless of age group and sex. A positive association between carotid plaque burden and adiposity was found (OR of having at least one plaque per SD in WHR 1.18 (95\% Cl 1.06 to 1.31) for men; 1.15 (1.06 to 1.25) for women)) adjusted for age, smoking and education in a pooled analysis of the two studies. There was little evidence of the interaction between study and adiposity. These effects did not differ between the two studies. However, neither adiposity nor CVD risk factors (smoking, systolic blood pressure, cholesterol, glycosylated haemoglobin) explained the higher carotid plaque burden in KYH compared with Troms $\emptyset 7$.

Conclusion Adiposity, especially abdominal adiposity, is a risk factor for carotid plaque in Russia and Norway, although neither adiposity nor established CVD risk factors explained the higher plaque burden in Russia. To reduce the CVD burden in Russia, beyond prevention and treatment of adiposity, further research is required to understand why Russia has a high burden of atherosclerosis.
Strengths and limitations of this study

This is the first study to compare adiposity level, carotid plaque burden and its association with adiposity between Russia and Western European country with low cardiovascular disease mortality.

- The use of two substantial population-based studies with similar study period and study protocols enabled us to make a direct comparison of two populations.

- Waist circumference was measured at different measurement sites between the two studies.

- We did not assess visceral adiposity or body composition.

\section{INTRODUCTION}

The mortality rate from cardiovascular disease (CVD) has been decreasing for many years in Western Europe, and more recently, in Eastern Europe. ${ }^{1}$ However, rates vary substantially between countries, with Russia having one of the highest CVD mortality rates, ${ }^{2}$ although it has been declining since 2005. ${ }^{3}$ In 2012-2016, the CVD mortality rate at working ages in Russia was eight times higher than that in Norway. ${ }^{4}$ These premature deaths contribute to the relatively low life expectancy for such an industrialised country. However, the reasons for this very high CVD burden in Russia remain unclear. ${ }^{4}$ It appears that the differences in conventional CVD risk factors such as smoking, blood pressure and cholesterol levels do not explain this difference well. ${ }^{56}$

The increase in obesity over the past decades is a growing concern worldwide, including in Russia and countries of Western 
Europe, ${ }^{7}$ and has an effect on mortality levels. ${ }^{8}$ In addition to general obesity, however, the extent of abdominal obesity is likely to be important as there is evidence that it is more strongly associated with CVD events than general adiposity assessed using body mass index (BMI). ${ }^{9-11}$ However, data on population levels of abdominal obesity (such as waist-to-hip ratio (WHR)) is far less common than for BMI, in Russia as well as in other countries. To the best of our knowledge, the contribution of general or abdominal obesity to the gap in CVD burden between Russia and Western European countries has not been investigated in spite of increasing importance of obesity as a CVD risk factor.

Carotid plaque, representing an advanced stage of atherosclerosis, is predictive of future CVD events. ${ }^{12}$ Carotid atherosclerosis may be easily and reliably detected using an ultrasound examination making carotid plaque a good surrogate marker of atherosclerotic CVD burden in large-scale epidemiological studies. Our previous study using Tromsø Study fifth survey has shown that abdominal adiposity was more closely associated with carotid plaque burden than BMI. ${ }^{13}$ Furthermore, WHR showed the larger effect size than waist circumference (WC) and waist to height ratio.

We used data from two studies from general populations with very different CVD mortality in Europe: Know Your Heart (KYH) study in Russia and Tromsø Study seventh survey (Tromsø 7) in Norway. Our aims were: (1) to compare general and abdominal adiposity levels, represented by BMI and WHR, respectively, and the burden of carotid plaque in Russia with those in Norway, a low CVD mortality country; (2) to investigate the association of BMI or WHR with carotid plaque in both populations and (3) to investigate whether BMI or WHR or other factors can explain difference in carotid plaque burden between the two populations.

\section{METHODS}

\section{Study design and participants}

We used data from two studies; the KYH study from Russia and the Troms $\varnothing 7$ study from Norway. Researchers from the two studies worked together at the design stage to align aspects of the study protocols used, including the detailed standard operating procedures for carotid ultrasound examinations as described elsewhere. ${ }^{414} 15$

$\mathrm{KYH}$ is a population-based cross-sectional study of 4500 women and men aged 35-69 years conducted between 2015 and 2017 in two Russian cities: Novosibirsk and Arkhangelsk. The details of KYH have been described elsewhere. ${ }^{4}$ Briefly, participants were recruited from a random sample of the population stratified by age and gender, derived from the list of the Territorial Health Insurance Funds. Trained interviewers visited the addresses on the list and identified residents of the target age and sex. Information on sociodemographic characteristics, CVD risk factors and medical history was collected using structured questionnaires completed on tablet computers. At the end of the interview, participants were invited to have a comprehensive examination including anthropometric measurement, blood sampling, and carotid ultrasound 1 or 2 weeks later. Response rates for initial interview were 53\% and $27 \%$ in Arkhangelsk and Novosibirsk, respectively. Of those interviewed $89 \%$ attended the subsequent medical examination. All participants of the medical examination provided signed informed consent.

The Troms $\varnothing$ Study is an ongoing population-based study in Tromsø municipality, North Norway and consists of seven surveys from 1974 to $2016 .{ }^{16}$ In the seventh wave (Troms $\varnothing 7$ ), all residents in Troms $\varnothing$ aged 40 years and older were invited to participate. The questionnaires were completed, a brief physical examination was carried out and biological samples were taken. A random sample, including previous participants, was invited to a second visit to undergo more comprehensive medical examinations. A total of 21083 attended the first visit and the response rate was $65 \%$. A total of 4153 participants were invited to a carotid ultrasound examination and 2974 $(71.6 \%)$ attended.

In the two studies, participants aged between 40 and 69 years $(n=5782)$ were eligible for the present study. We excluded participants with missing data on all adiposity measures $(n=42)$, and potential confounders and mediators $(\mathrm{n}=678)$, leaving 3262 participants from $\mathrm{KYH}(57 \%$ women) and 1800 from Troms $\varnothing 7$ (55\% women) for the analyses.

\section{Assessment of anthropometric measures and other CVD risk factors}

In both studies, height and weight were assessed by trained staff using standard methods (see online supplementary material). BMI was calculated by dividing weight in kilograms by squared height in metres. WC was measured at a different site in the two studies: in KYH WC was measured at the narrowest part of the trunk to the nearest millimetre using a tape measure while in Troms $\varnothing 7 \mathrm{WC}$ was measured at the level of the umbilicus. Hip circumference was measured at the widest part in both studies. To ensure WC was comparable between the two studies, WC in Troms $\varnothing 7$ was converted to the narrowest waist using a conversion equation by Mason and Katzmarzyk. ${ }^{17}$ Among anthropometric measures of abdominal adiposity WHR was selected because it has been found to be strongly associated with CVD events. ${ }^{10} 1118$

Information on age (5-year categories), smoking (current smoker, ex-smoker, never-smoker), educational attainment (higher education: yes/no) and medical history of diabetes mellitus (DM) (yes/no) were collected through face-to-face interview in $\mathrm{KYH}$ and selfadministered questionnaire in Troms $\varnothing$ 7. The assessment of systolic blood pressure (SBP) and other laboratory data are described elsewhere (see online supplementary material). ${ }^{4}$ 


\section{Ultrasound examination}

Technical details of the examination protocols have been described elsewhere. ${ }^{41415}$ Briefly, both carotid arteries were scanned for carotid plaques in the common carotid artery (CCA), bifurcation and internal carotid artery (ICA) using a Vivid Q (GE Health care) with $6 \sim 13 \mathrm{MHz}$ linear transducers in KYH and Vivid 7 (GE Health care) with a linear $12 \mathrm{MHz}$ transducer in Troms $\varnothing 7$ (see online supplementary material). Carotid plaque was defined according to the Mannheim Consensus as a focal structure encroaching into the arterial lumen by at least $0.5 \mathrm{~mm}$, or having a thickness $\geq 50 \%$ greater than the surrounding intima-media thickness (IMT), or IMT $>1.5$ $\mathrm{mm}$ as measured from the media-adventitia interface to the intima-lumen interface. ${ }^{19}$

To evaluate the burden of carotid plaque, we created a cumulative plaque score by assigning a score of one for the presence of one or more plaques in each of the six carotid segments (CCA, bifurcation and ICA of each carotid artery) with a maximum possible score of 6 for each individual.

\section{Statistical methods}

Analyses were conducted stratifying by sex a priori. Two outcome measures were used: the presence of plaques as a binary outcome and plaque score. As exposures, BMI and WHR were used to represent general and abdominal adiposity, respectively. To enable direct comparison of the magnitude of the effects of BMI and WHR, sex-specific adiposity z-scores standardised to Troms $\varnothing 7$ participants were created by subtracting the mean and dividing by the SD of each measure in Tromsø 7.

Variables included in the model were selected from established CVD risk factors. ${ }^{20}$ Age, smoking and education were considered a priori confounders while SBP, high-density lipoprotein cholesterol, low-density lipoprotein cholesterol, glycosylated haemoglobin (HbAlc) and medical history of DM were considered as potential mediators. Sex-specific linear and logistic regression models were used to investigate the associations of each adiposity with plaque score and presence of plaques respectively. A series of models were fitted that were specific to each sex and study (four in all). Model 1 adjusted for age (5-year age groups). Model 2, our main model to elucidate the association between adiposity and plaque burden, further adjusted for potential confounders. Model 3 further adjusted for potential mediators to see to what extent the association was mediated by these factors. These analyses were conducted using the data from each study and the pooled data from the two studies after checking for interaction with study. This was done by adding an interaction term between study and adiposity using pooled data: testing for statistical significance using likelihood ratio tests for logistic regression and Wald tests for linear regression.

Finally, to estimate the difference in plaque burden between the two studies, we applied a similar set of models as already described to the pooled data using a binary indicator for study. The associations between each study and plaque burden (the presence of plaques, plaque score) were estimated using logistic and linear regression, respectively. To look at adjusted difference in plaque burden between the two studies, three similar models adjusted for age, confounders and mediators, were applied without adjustment for adiposity. We then separately added each adiposity measure to these models to estimate the effect of adiposity on between-study difference in carotid plaque burden.

STATA V.15 (StataCorp) was used for all the analyses.

\section{Patient and public involvement}

This study was part of the International Project on Cardiovascular Disease in Russia (IPCDR). IPCDR had an important Public Engagement component as described on the project website (https://knowyourheart.science/). This involved a wide range of activities that ranged from television programmes on the KYH study, focus groups and publication of popular articles on CVD in the Russian media. The Heart to Heart comparisons of Norway with Russia have received media coverage in the Norwegian media, and the Troms $\varnothing 7$ study itself involved extensive publicity engagement with the citizens of the city of Troms $\emptyset$. Members of the general public were not involved in the design or the study or its scientific aims.

\section{RESULTS}

\section{Baseline characteristics of participants}

Table 1 shows participants' baseline characteristics. The age-adjusted prevalence of current smoking in men was much higher in KYH than Troms $\varnothing 7$ but similar for women. However, female never smokers made up twothirds in KYH but just over a third in Tromsø 7. Mean SBP was considerably higher in KYH than in Troms $\varnothing 7$.

\section{Adiposity}

Both BMI and WHR were higher for women in KYH than those in Tromsø 7. Adiposity z-scores for BMI and WHR for the KYH women standardised to the Tromsø 7 population adjusted for age were 0.58 (95\% CI 0.49 to 0.68 ) and 0.84 (95\% CI 0.76 to 0.92 ), respectively. However, adiposity did not differ between men in the two studies.

\section{Prevalence of carotid plaques}

The prevalence of carotid plaques and the mean plaque score increased with age in both women and men (table 2). The burden of plaques was consistently higher in $\mathrm{KYH}$ than Troms $\varnothing$ in both sexes.

The association between carotid plaque burden and adiposity: a pooled analysis of the two studies

Table 3 shows the ORs for having at least one carotid plaque per 1SD increase in each adiposity measure by sex from the pooled analysis. We also analysed the two studies separately, but only presented the result from the pooled data based on the test of interaction described below. The two adiposity measures were not adjusted for each other. 
Table 1 Participant characteristics in Know Your Heart (KYH) and Tromsø 7 (T7) ${ }^{\star}$

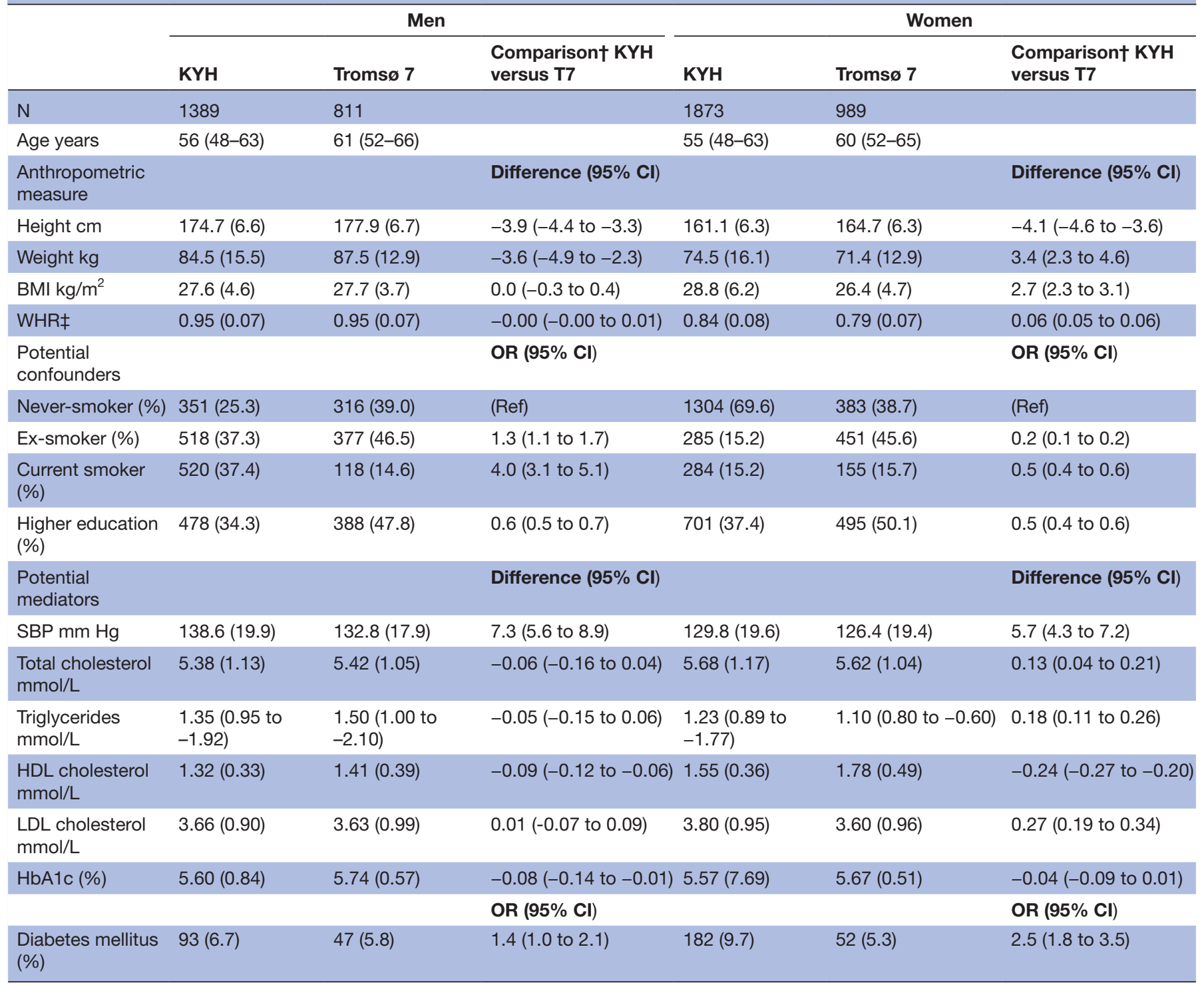

Data are presented as percentages for binary variables and as mean values (SD) continuous variables, except for age, triglycerides which are presented as median (IQR).

*Analyses were restricted to participants aged between 40 and 69 years with information on all covariates.

†All comparisons age adjusted.

ҒWC in Tromsø 7 assessed at the level of the umbilicus was converted to the narrowest WC so that it can be comparable with WC in KYH. WHR in T7 are calculated using converted WC.

.BMI, body mass index; HbA1c, glycosylated haemoglobin; HDL, high-density lipoprotein; LDL, low-density lipoprotein; SBP, systolic blood pressure; WC, waist circumference; WHR, waist-to-hip ratio.

After adjustment for confounders (model 2), there was evidence of association between all adiposity measures and the presence of plaques except for BMI in women. WHR showed larger standardised ORs (women $1.1595 \%$ CI 1.06 to 1.25 , men $1.1895 \%$ CI 1.06 to 1.31 ) than BMI. After further adjustment for cardiometabolic mediators (model 3), all ORs decreased substantially.

Table 4 shows the difference in plaque score per $1 \mathrm{SD}$ increase in each adiposity measure. In women, adiposity was associated with an increase in plaque score. Again, WHR showed a larger effect size (increase per 1SD change $0.10995 \%$ CI 0.070 to 0.147 ) than BMI. Additional adjustments for cardiometabolic mediators reduced both effect sizes substantially. For men, there was no evidence of an association of BMI and WHR with plaque score.

Tests for interaction between study and adiposity were not statistically significant except for the association between BMI and the presence of plaque in women and that between WHR and the plaque score in women, suggesting that there is little evidence that the association between adiposity and plaque burden differs between the two studies (online supplementary table 1, online supplementary figure $1 \mathrm{~A}, \mathrm{~B}$ ). 
Table 2 The prevalence of carotid plaques and plaque score according to study and sex

\begin{tabular}{|c|c|c|c|c|c|c|}
\hline & \multicolumn{3}{|c|}{ Men } & \multicolumn{3}{|c|}{ Women } \\
\hline & KYH & Tromsø 7 & $\begin{array}{l}\text { Comparison } \\
\text { KYH versus T7* }\end{array}$ & KYH & Tromsø 7 & $\begin{array}{l}\text { Comparison } \\
\text { KYH versus T7* }\end{array}$ \\
\hline $\begin{array}{l}\text { Prevalence n } \\
(\%)\end{array}$ & & & OR $(95 \% \mathrm{Cl})$ & & & OR (95\% Cl) \\
\hline All age & 1050 (75.6) & $499(61.5)$ & 3.2 (2.6 to 4.0$)$ & 1043 (55.7) & $478(48.3)$ & 1.9 (1.6 to 2.3 ) \\
\hline $50-59$ & $340 / 450(75.6)$ & $104 / 191(54.5)$ & 2.7 (1.9 to 4.0$)$ & $328 / 605$ (54.2) & $112 / 273(41.0)$ & 1.7 (1.3 to 2.3 ) \\
\hline $60-69$ & 498/545 (91.4) & $357 / 472(75.6)$ & 3.5 (2.4 to 5.1$)$ & $541 / 698(77.5)$ & $326 / 527$ (61.9) & 2.1 (1.7 to 2.7$)$ \\
\hline $\begin{array}{l}\text { Plaque score } \\
\text { mean (SD) }\end{array}$ & & & $\begin{array}{l}\text { Difference }(95 \% \\
\text { Cl) }\end{array}$ & & & $\begin{array}{l}\text { Difference }(95 \% \\
\text { CI) }\end{array}$ \\
\hline $60-69$ & $2.7(1.6)$ & $1.5(1.2)$ & $1.2(1.1$ to 1.4$)$ & $1.7(1.4)$ & $1.1(1.0)$ & 0.7 (0.5 to 0.8$)$ \\
\hline
\end{tabular}

${ }^{*}$ Adjusted for categorical age (5-year interval).

$\mathrm{KYH}$, Know Your Heart.

\section{Between-study differences in carotid plaque burden and the effect of adiposity}

Figure 1 compares the carotid plaque burden between the two studies with and without adjustment for adiposity. Without adjustment for adiposity measures, the OR for having at least one plaque in KYH compared with Troms $\varnothing$ 7 was 1.97 (95\% CI 1.62 to 2.38) in women and 2.78 (95\% CI 2.21 to 3.49 ) in men (figure 1A, online supplementary table 2A model 2). Further adjustment for BMI or WHR separately had only a small effect on this OR for both men and women (figure 1A). The between-study difference remained large and statistically significant after further adjustment for cardiometabolic mediators (figure 1A, online supplementary table 2A model 3 ).

Similarly, without adjustment for adiposity, participants in KYH had a higher mean plaque score than those in Troms $\varnothing 7$ by 0.51 (95\% CI 0.42 to 0.60 ) for women and 0.89 (95\% CI 0.77 to 1.00) for men; these estimates decreased slightly for women and hardly changed at all for men with further adjustment for adiposity (figure 1B, online supplementary table 2B). The between-study difference remained significant after further adjustment for cardiometabolic mediators (figure 1B, online supplementary table 2B model 3).

\section{DISCUSSION}

There was evidence of positive associations between adiposity, especially abdominal adiposity, and carotid plaque burden, but no convincing evidence that the strength of these associations differed between the two studies. These associations were largely mediated by cardio-metabolic CVD risk factors. However, neither adiposity nor the confounders and potential mediators explained the substantially greater burden of plaque in the KYH study in Russia compared with the Tromsø 7

Table 3 ORs for having at least one plaque per 1 SD increase in each adiposity measure: pooled results from the two studies

\begin{tabular}{|c|c|c|c|c|c|c|}
\hline & Model 1 OR $(95 \% \mathrm{Cl})$ & $P$ value & Model 2 OR (95\% Cl) & $P$ value & Model 3 OR $(95 \% \mathrm{Cl})$ & P value \\
\hline \multicolumn{7}{|c|}{ Men $(n=2200)$} \\
\hline st BMI & 1.12 (1.02 to 1.23$)$ & 0.02 & 1.13 (1.03 to 1.24$)$ & 0.01 & 1.01 (0.91 to 1.12$)$ & 0.83 \\
\hline \multicolumn{7}{|c|}{ Women (n=2862) } \\
\hline st BMI & 1.06 (0.99 to 1.14$)$ & 0.08 & 1.06 (0.99 to 1.13$)$ & 0.09 & 0.94 (0.87 to 1.01$)$ & 0.08 \\
\hline
\end{tabular}

Model 1: adjusted for categorical age (5 years) and study, model 2: adjust for variables in model 1 plus potential confounders (smoking and education), model 3: adjusted for variables in model 2 plus potential mediators (systolic blood pressure, HDL cholesterol, LDL cholesterol, glycated haemoglobin, medical history of diabetes).

HDL, high-density lipoprotein; LDL, low-density lipoprotein; St BMI, body mass index z-score; st WHR, waist-to-hip ratio z-score. 
Table 4 Difference in plaque score per 1 SD increase in each adiposity measure: pooled results from the two studies

\begin{tabular}{|c|c|c|c|c|c|c|}
\hline & Model 1 slope $(95 \% \mathrm{Cl})$ & $P$ value & Model 2 slope (95\% Cl) & $P$ value & Model 3 slope $(95 \% \mathrm{Cl})$ & $P$ value \\
\hline \multicolumn{7}{|c|}{ Men $(n=2200)$} \\
\hline st BMI & $-0.021(-0.069$ to 0.026$)$ & 0.38 & $-0.008(-0.055$ to 0.039$)$ & 0.74 & $-0.091(-0.143$ to -0.040$)$ & $<0.001$ \\
\hline \multicolumn{7}{|c|}{ Women (n=2862) } \\
\hline st BMl & $0.023(-0.009$ to 0.056$)$ & 0.16 & $0.023(-0.010$ to 0.055$)$ & 0.17 & $-0.056(-0.091$ to -0.021$)$ & 0.002 \\
\hline
\end{tabular}

Model 1: adjusted for categorical age (5 years) and study, model 2: adjust for variables in model 1 plus potential confounders (smoking and education), model 3: adjusted for variables in model 2 plus potential mediators (systolic blood pressure, HDL cholesterol, LDL cholesterol, glycated haemoglobin, medical history of diabetes.

HDL, high-density lipoprotein; LDL, low-density lipoprotein; St BMI, body mass index z-score; st WHR, waist-to-hip ratio z-score.

study in Norway. To the best of our knowledge, this is the first study to directly investigate the role of adiposity in high CVD burden in a general population in Russia in comparison with another country.
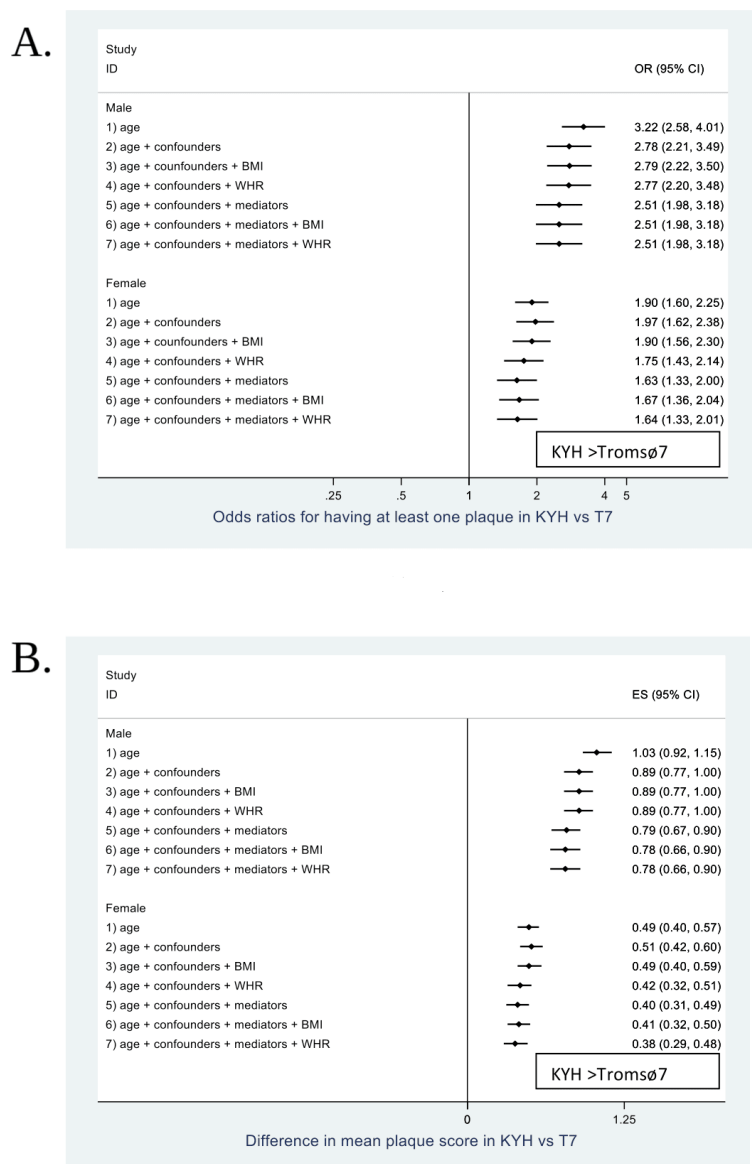

Figure 1 (A) ORs $(95 \% \mathrm{Cl})$ for having at least one plaque in $\mathrm{KYH}$ versus Troms $\varnothing 7$ with and without adjustment for adiposity. (B) Differences $(95 \% \mathrm{Cl})$ in the mean plaque score in KYH compared with Tromsø 7 with and without adjustment for adiposity. Confounders: smoking and education, mediators: systolic blood pressure, HDL cholesterol, LDL cholesterol, glycated haemoglobin, medical history of diabetes. BMI, body mass index; HDL, highdensity lipoprotein; KYH, Know Your Heart; LDL, low-density lipoprotein; WHR, waist-to-hip ratio.
Given the similar adiposity level between KYH and Troms $\varnothing 7$ among men, it is not surprising that higher plaque burden in men in $\mathrm{KYH}$ is not explained by adiposity. However, even among women whose adiposity level was considerably higher in KYH than Tromsø 7 , the adjustment for adiposity had little impact on the interstudy difference in carotid plaque burden. Furthermore, additional adjustment for CVD and metabolic risk factors such as smoking, SBP and cholesterol level slightly reduced this interstudy difference, but the betweenstudy difference remained for both men and women, suggesting that there are other determinants of higher carotid plaque burden in a population in $\mathrm{KYH}$. This is consistent with previous studies conducted 20 or more years ago, showing that differences in traditional CVD risk factors did not fully explain the high CVD burden in Russia compared with Western European countries. ${ }^{56}$ More advanced subclinical atherosclerosis in participants in KYH compared with Troms $\varnothing 7$ is in keeping with the higher CVD mortality rate in Russia than Western European countries. $^{421}$

The development of coronary artery disease and atherosclerotic plaque is a gradual process that occurs across the life course. ${ }^{22}$ The extent to which single cross-sectional measurements of risk factors such as blood pressure and smoking can capture the full impact of these risk factors on the burden of carotid plaque is therefore questionable. In making the sort of comparisons between populations that are the focus of this paper, therefore, it could be that we are underestimating the potential contribution of these risk factors to differences in plaque burden, particularly if risk factor profiles have been changing.

One potential determinant of high CVD risk in Russia that we have not included is alcohol which has been shown to be related to mortality from CVD ${ }^{23}$ Vikhireva $e t$ $a l$ added hazardous alcohol consumption to the high-risk version of Systematic COronary Risk Evaluation (SCORE) to see whether this modified model improved prognostic performance of SCORE for future CVD events in the Russian population. However, this modification did not improve the prediction of CVD events ${ }^{24}$ although the study had limited follow-up and relatively small numbers 
of events. Moreover, it excluded as an outcome alcoholic cardiomyopathy that contributes to the high CVD mortality in Russia ${ }^{25}$ involving processes other than atherosclerosis. ${ }^{26}$ Differences in treatment and access to the medical facilities between Western Europe and Russia is likely to partly account for the higher CVD mortality rates in Russia, but it is unlikely that differences in treatment could account for the differences in subclinical atherosclerosis in a population-based samples. Furthermore, the treatment and access to appropriate medical care have been improving rapidly in Russia, especially in large cities, so this is likely to be a less important factor in the future. ${ }^{27} 28$ Identification of the determinant(s) of advanced subclinical atherosclerosis in Russia will be important to target interventions to reduce CVD burden.

There was evidence of positive associations between adiposity and plaque burden in both studies emphasising the importance of the control of adiposity, especially abdominal adiposity, to curb the CVD burden in both countries. The prevalence of obesity in Russia has been increasing, ${ }^{7}$ with the notably high level among women being of particular concern. ${ }^{7}$ Another important implication of our findings is the importance of the control of cardiovascular and cardiometabolic mediators. The associations between adiposity and carotid plaque burden were largely mediated by SBP, cholesterol level and HbAlc. The effective control of these traditional risk factors will mitigate the negative effect of adiposity.

This study is the first, to our knowledge, to bring together data from a high and relatively low CVD mortality country to investigate the association of adiposity with carotid plaque, and also the extent to which this can explain differences in the burden of carotid plaque between the two populations. Moreover, this was done using ultrasound examination protocols that were aligned between the two studies. No previous studies have compared imaging of atherosclerotic changes in general populations between Russia and Western countries. However, our investigation has some limitations. First, the anthropometric measures we used are crude measures of visceral adiposity. However, estimation of visceral adipose tissue using MRI and CT is resource demanding and logistically difficult in large epidemiological studies. Second, WC was measured differently between the two studies. Although the conversion of WC was made using a conversion equation, this did not allow for individual variability. Standardisation of the protocol of WC measurement would be important in future studies. Third, we did not include alcohol in our regression models, although it is likely to play an important role in CVD mortality in Russia. ${ }^{29-32}$ This is because alcohol consumption in the two study populations was not directly comparable. Finally, as always, caution must be exercised in generalising to the national situation the results we have obtained from the two cross-sectional studies of selected groups in two cities in Russian and one in Norway city.

Overall, our findings have two implications with respect to tackling the high CVD burden in Russia. First, although adiposity failed to explain higher plaque burden in Russia compared with Norway, adiposity, especially abdominal adiposity, appeared to contribute to an increase in carotid plaque burden through cardiometabolic mediators such as blood pressure and cholesterol. The reduction of adiposity level will be important to avoid further CVD burden in addition to the control of cardiometabolic mediators. Second, our findings suggest that there are other unidentified risk factors that determine the higher carotid plaque burden in Russia compared with Norway. Further studies will be needed to identify them.

\section{Author affiliations}

${ }^{1}$ London School of Hygiene and Tropical Medicine, London, UK

${ }^{2} U \mathrm{UTT}$ The Arctic University of Norway, Troms $\emptyset$, Norway

${ }^{3}$ Reserach Institute of Internal and Preventive Medicine, Novosibirsk, Russian

Federation

${ }^{4}$ Novosibirsk State Medical University, Novosibirsk, Russian Federation

${ }^{5}$ Northern State Medical University, Arkhangelsk, Russian Federation

${ }^{6}$ University College London, London, UK

Contributors $\mathrm{YI}, \mathrm{CF}$ and DL made substantial contributions to the conception and design of the work. EM, LAH, AR, AVK, MK and SM were centrally involved in the design of the Know Your Heart and/or the conduct of the associated fieldwork. YI undertook the analysis of the data with the regular input of $\mathrm{CF}, \mathrm{AH}$ and DL.YI wrote the first drafts of the manuscript (including the interpretation of the results) which was then refined over successive versions by Yl with the input of all authors. All authors approved the submitted version. All authors agreed both to be personally accountable for their own contributions and to ensure that questions related to the accuracy or integrity of any part of the work, even ones in which the author was not personally involved, were appropriately investigated, resolved and the resolution documented in the paper.

Funding The Know Your Heart study is a component of the International Project on Cardiovascular Disease in Russia (IPCDR). IPCDR was supported by the a Wellcome Trust Strategic Award (100 217), funds from UiT The Arctic University of Norway, Norwegian Institute of Public Health, and the Norwegian Ministry of Health and Social Affairs.

Disclaimer The funders had no role in study design, data collection and analysis, decision to publish, or preparation of the manuscript.

Competing interests None declared.

Patient consent for publication Not required.

Ethics approval Ethical approval for the KYH study was received from the ethics committees of the London School of Hygiene \& Tropical Medicine (approval number 8808 received 24/02/2015); Novosibirsk State Medical University (approval number 75 received 21/05/2015); the Institute of Internal and Preventative Medicine (approval received 26/12/2014), Novosibirsk and the Northern State Medical University, Arkhangelsk (approval number 01/01-15 received 27 January 2015). Ethical approval for Troms $\emptyset 7$ was obtained the Regional Committee for Medical and Health Research Ethics (reference number 2014/940).

Provenance and peer review Not commissioned; externally peer reviewed.

Data availability statement Data are available on reasonable request. Data availability statement: Metadata is available for the Tromsø study at http:// tromsoundersokelsen.uit.no/tromso/ and for the Know Your Heart study at https:// metadata.knowyourheart.science/.The data used in these analyses cannot be placed in the public domain because of ethical and data protection restrictions.

Open access This is an open access article distributed in accordance with the Creative Commons Attribution Non Commercial (CC BY-NC 4.0) license, which permits others to distribute, remix, adapt, build upon this work noncommercially, and license their derivative works on different terms, provided the original work is properly cited, appropriate credit is given, any changes made indicated, and the use is non-commercial. See: http://creativecommons.org/ licenses/by-nc/4.0/.

ORCID iD

Yume Imahori http://orcid.org/0000-0002-2176-7150 


\section{REFERENCES}

1 Ezzati M, Obermeyer Z, Tzoulaki I, et al. Contributions of risk factors and medical care to cardiovascular mortality trends. Nat Rev Cardiol 2015;12:508-30.

2 Timmis A, Townsend N, Gale C, et al. European Society of cardiology: cardiovascular disease statistics 2017. Eur Heart $J$ 2018;39:508-79.

3 Grigoriev P, Meslé F, Shkolnikov VM, et al. The recent mortality decline in Russia: beginning of the cardiovascular revolution? Popul Dev Rev 2014;40:107-29.

4 Cook S, Malyutina S, Kudryavtsev AV, et al. Know your heart: rationale, design and conduct of a cross-sectional study of cardiovascular structure, function and risk factors in 4500 men and women aged 35-69 years from two Russian cities, 2015-18. Wellcome Open Res 2018;3:67.

5 Kuulasmaa K, Tunstall-Pedoe H, Dobson A, et al. Estimation of contribution of changes in classic risk factors to trends in coronaryevent rates across the who MONICA project populations. Lancet 2000;355:675-87.

6 Averina M, Nilssen O, Brenn T, et al. High cardiovascular mortality in Russia cannot be explained by the classical risk factors. The Arkhangelsk Study 2000. Eur J Epidemiol 2003;18:871-8.

7 NCD Risk Factor Collaboration (NCD-RisC). Trends in adult bodymass index in 200 countries from 1975 to 2014: a pooled analysis of 1698 population-based measurement studies with $19 \cdot 2$ million participants. Lancet 2016;387:1377-96.

8 Vidra N, Trias-Llimós S, Janssen F. Impact of obesity on life expectancy among different European countries: secondary analysis of population-level data over the 1975-2012 period. BMJ Open 2019;9:e028086.

9 Peters SAE, Bots SH, Woodward M. Sex differences in the association between measures of general and central adiposity and the risk of myocardial infarction: results from the UK Biobank. J Am Heart Assoc 2018;7. doi:10.1161/JAHA.117.008507. [Epub ahead of print: 28 Feb 2018].

10 Emdin CA, Khera AV, Natarajan P, et al. Genetic association of Waist-to-Hip ratio with cardiometabolic traits, type 2 diabetes, and coronary heart disease. JAMA 2017;317:626-34.

11 Dale CE, Fatemifar G, Palmer TM, et al. Causal associations of adiposity and body fat distribution with coronary heart disease, stroke subtypes, and type 2 diabetes mellitus: a Mendelian randomization analysis. Circulation 2017;135:2373-88.

12 Inaba Y, Chen JA, Bergmann SR. Carotid plaque, compared with carotid intima-media thickness, more accurately predicts coronary artery disease events: a meta-analysis. Atherosclerosis 2012;220:128-33.

13 Imahori Y, Mathiesen EB, Leon DA, et al. The contribution of obesity to carotid atherosclerotic plaque burden in a general population sample in Norway: the Troms $\varnothing$ study. Atherosclerosis 2018;273:15-20.

14 Fosse E, Johnsen SH, Stensland-Bugge E, et al. Repeated visual and computer-assisted carotid plaque characterization in a longitudinal population-based ultrasound study: the Troms $\varnothing$ study. Ultrasound Med Biol 2006;32:3-11.

15 Imahori Y. Adiposity and carotid atherosclerosis in two populations at very different risk of cardiovascular mortality, 2019. Available: https:// researchonline.Ishtm.ac.uk/id/eprint/4654524/
16 Jacobsen BK, Eggen AE, Mathiesen EB, et al. Cohort profile: the Tromso study. Int J Epidemiol 2012;41:961-7.

17 Mason C, Katzmarzyk PT. Variability in waist circumference measurements according to anatomic measurement site. Obesity 2009;17:1789-95.

18 O'Donnell MJ, Chin SL, Rangarajan S, et al. Global and regional effects of potentially modifiable risk factors associated with acute stroke in 32 countries (INTERSTROKE): a case-control study. Lancet 2016;388:761-75.

19 Touboul P-J, Hennerici MG, Meairs S, et al. Mannheim carotid intima-media thickness and plaque consensus (2004-20062011). An update on behalf of the Advisory Board of the 3rd, 4th and 5 th watching the risk symposia, at the 13th, 15th and 20th European stroke conferences, Mannheim, Germany, 2004, Brussels, Belgium, 2006, and Hamburg, Germany, 2011. Cerebrovasc Dis 2012;34:290-6

20 Lloyd-Jones DM, Hong Y, Labarthe D, et al. Defining and setting national goals for cardiovascular health promotion and disease reduction: the American heart association's strategic impact goal through 2020 and beyond. Circulation 2010;121:586-613.

21 Townsend N, Wilson L, Bhatnagar P, et al. Cardiovascular disease in Europe: epidemiological update 2016. Eur Heart J 2016;37:3232-45.

22 Rose G. Incubation period of coronary heart disease. $\mathrm{Br} \mathrm{Med} \mathrm{J}$ 1982;284:1600-1.

23 Leon DA, Shkolnikov VM, McKee M, et al. Alcohol increases circulatory disease mortality in Russia: acute and chronic effects or misattribution of cause? Int J Epidemiol 2010;39:1279-90.

24 Vikhireva O, Kubinova R, Malyutina S, et al. Inclusion of hazardous drinking does not improve the score performance in men from central and eastern Europe: the findings from the HAPIEE cohorts. BMC Public Health 2014;14:1187.

25 Manthey J, Probst C, Rylett M, et al. National, regional and global mortality due to alcoholic cardiomyopathy in 2015 . Heart 2018;104:1663-9.

26 Leon DA, Shkolnikov VM, Borinskaya S, et al. Hazardous alcohol consumption is associated with increased levels of B-type natriuretic peptide: evidence from two population-based studies. Eur J Epidemiol 2013;28:393-404.

27 Timonin S, Kontsevaya A, McKee M, et al. Reducing geographic inequalities in access times for acute treatment of myocardial infarction in a large country: the example of Russia. Int J Epidemiol 2018:47:1594-602

28 Kontsevaya A, Sabgaida T, Ivanova A, et al. How has the management of acute coronary syndrome changed in the Russian Federation during the last 10 years? Health Policy 2017;121:1274-9.

29 GBD 2016 Russia Collaborators. The burden of disease in Russia from 1980 to 2016: a systematic analysis for the global burden of disease study 2016. Lancet 2018;392:1138-46.

30 Feigin VL, Roth GA, Naghavi M, et al. Global burden of stroke and risk factors in 188 countries, during 1990-2013: a systematic analysis for the global burden of disease study 2013. Lancet Neurol 2016;15:913-24.

31 Zaridze D, Brennan P, Boreham J, et al. Alcohol and cause-specific mortality in Russia: a retrospective case-control study of 48,557 adult deaths. Lancet 2009;373:2201-14.

32 Leon DA, Saburova L, Tomkins S, et al. Hazardous alcohol drinking and premature mortality in Russia: a population based case-control study. Lancet 2007;369:2001-9. 